\title{
Opening of a Gap in Graphene Due to Supercell Potential: Group Theory Point of View
}

\author{
E. Kogan, M. Kaveh \\ Department of Physics, Bar-Ilan University, Ramat-Gan, Israel \\ Email: Eugene.Kogan@biu.ac.il, Moshe.Kaveh@biu.ac.il
}

Received 25 February 2014; revised 22 March 2014; accepted 17 April 2014

Copyright $@ 2014$ by authors and Scientific Research Publishing Inc.

This work is licensed under the Creative Commons Attribution International License (CC BY).

http://creativecommons.org/licenses/by/4.0/

(c) (i) Open Access

\begin{abstract}
We analyze in the framework of the space group theory the change of the dispersion law in grapenein and the vicinity of the (former) Dirac points due to application of supercell potential with the $\sqrt{3} \times \sqrt{3}$ space priodicity and the same point symmetry as graphene.
\end{abstract}

\section{Keywords}

\section{Graphene, Group Theory, Space Groups}

\section{Introduction}

Graphene is a two-dimensional crystal of carbon atoms, which form a honeycomb lattice with the point symmetry described by the group $D_{6 h}$. The first Brillouin zone (BZ) has a hexagonal form, and the conduction band touches the valence band in six BZ corners which form two non-equivalent triads of BZ corners, $\boldsymbol{K}$ and $\boldsymbol{K}^{\prime}$. One of the routes toward tailoring the electronic properties of graphene is through the adsorption of metals [1] [2]. Recently, several types of adatoms were used to dope graphene in attempts to tailor properties of graphenebased devices [3]-[7]. The gap opening in the high symmetry points for the hexagonal lattices due to interaction with the interface was considered in [8]. In Ref. [9], it was shown that, using $a b$ initio density functional ory calculations, the adsorption of an alkali-metal submonolayer ongraphene occupying every third hexagon of the honeycomb lattice in a commensurate $(\sqrt{3} \times \sqrt{3}) R 30^{\circ}$ arrangement induces an energy gap in the spectrum of graphene. We decided to analyze this opening of the gap in the framework of the group theory. In our previous publications [10]-[12] we summed up the classification of the energy bands in graphene on the basis of the point 
group analysis. The only fact from that analysis we need in the present work, is the fact that the little group at the point $\boldsymbol{K}$ is $D_{3 h}$ and the bands $\pi$ and $\pi^{*}$ realize $E^{\prime \prime}$ representation of the group. The same can be said about the point $\boldsymbol{K}^{\prime}$. This fact by itself means that the bands $\pi$ and $\pi^{*}$ touch each other at the points $\boldsymbol{K}$ and $\boldsymbol{K}^{\prime}$, and the electron states in the vicinity of these points are described by massless Dirac equation [11].

For the purpose of the present paper, we should put the abovementioned fact into the framework of the theory of the space group symmetry [13] [14], from which we will need only a few basic ideas. According to the theory of the space group symmetry, the bands $\pi$ and $\pi^{*}$ should be considered at the points $\boldsymbol{K}$ and $\boldsymbol{K}^{\prime}$ (these two points can be considered as the stars of the wave vector $\boldsymbol{K}$, and designated ${ }^{*} \boldsymbol{K}$ together, thus realizing a 4-dimensional representation of the $D_{6 h}$ group. Due to the identity

$$
D_{6 h}=D_{3 h} \cup C_{2} \times D_{3 h}
$$

any element of the group $D_{6 h}$ can be presented as an element of the group $D_{3 h} G$, or as a product of $C_{2}$ and such element. Representation of the point group $D_{3 h} E^{\prime \prime}$ realized at the point $\boldsymbol{K}\left(\boldsymbol{K}^{\prime}\right)$ defines representation of the space group realized at ${ }^{*} \boldsymbol{K}$. The matrix representing an element $G$ is a super-matrix $2 \times 2$

$$
D^{*} K, E^{\prime \prime}(G)=\left(\begin{array}{c|c}
D^{E^{\prime \prime}}(G) & 0 \\
\hline 0 & D^{E^{\prime \prime}}(G)
\end{array}\right)
$$

super-indices 1 and 2 referring to the points $\boldsymbol{K}$ and $\boldsymbol{K}^{\prime}$ respectively. The matrix representing an element $C_{2} \times G$ is

$$
D^{* K, E "}\left(C_{2} \times G\right)=\left(\begin{array}{c|c}
0 & \cdots \\
\cdots & 0
\end{array}\right) .
$$

We will not need the exact form of the non-diagonal matrix elements in Equation (3); what we need is the fact that the trace of the matrix $D^{* K, E^{\prime \prime}}\left(C_{2} \times G\right)$ is equal to zero. Naturally, when we consider dispersion in grapheme as it is, space group symmetry point of view adds very little in comparison to point group symmetry point of view, because the Hamiltonian, which has the symmetry $D_{3 h}$, is block-diagonal.

Now we apply the group theory to analyze what happens at the points $\boldsymbol{K}, \boldsymbol{K}^{\prime}$ in grapheme with a perfectly commensurate superlattice potential (which appears either because of the substrate or because of the absorbed atoms), which has the same point symmetry $D_{6 h}$ as graphene. We consider explicitly a $\sqrt{3} \times \sqrt{3}$ superlattice, known as the Kekule distortion of the honeycomb lattice [9]. In this case we may consider the Bril-louine zone (BZ) of the superlattice as the folding of the original [9] [15]. The folding leads to the identification of the corners of the original BZ ( $\boldsymbol{K}$ and $\boldsymbol{K}^{\prime}$ ) with the center $\tilde{\Gamma}$ of the new BZ. The Hamiltonian is no longer block diagonal and, because the points $\boldsymbol{K}$ and $\boldsymbol{K}^{\prime}$ are now identical, has the full symmetry $D_{6 h}$. We thus observe a paradox situation: due to decrease of the translational symmetry the point symmetry of the Hamiltonian has increased.

Because of the symmetry of the Hamiltonian, we need to decompose representation realized by Matrices (2) and (3) with respect to the irreducible representations of the group $D_{6 h}$.To obtain the decomposition, it is convenient to use equation

$$
a_{\alpha}=\frac{1}{g} \sum_{G} \chi(G) \chi_{\alpha}^{*}(G)
$$

which shows how many times a given irreducible representation $\alpha$ is contained in a reducible one [16]. In Equation (4) $g$ is the number of elements in the group, $\chi_{\alpha}(G)$ is the character of an operator $G$ in the irreducible representation $\alpha$ and $\chi(G)$ is the character of the operator $G$ in the representation being decomposed. Actually, even without using Equation (4), just by inspection of the two lowest line of Table 1 we obtain the decomposition

$$
R=E_{1 g}+E_{2 u} .
$$

We see that due to supercell potential two degenerate Dirac points disappear. At the point $\tilde{\Gamma}$ we have two merging bands realizing representation $E_{1 g}$ and another two merging bands realizing representation $E_{2 u}$. We 
Table 1. Characters table for irreducible representations of $D_{6}, D_{3 h}$ point groups.

\begin{tabular}{cccccccc}
\hline$D_{6}$ & & $E$ & $C_{2}$ & $2 C_{3}$ & $2 C_{6}$ & $3 U_{2}$ & $3 U_{2}^{\prime}$ \\
\hline$A_{1}$ & $D_{3 h}$ & $E$ & $\sigma$ & $2 C_{3}$ & $2 S_{3}$ & $3 U_{2}$ & $3 \sigma_{v}$ \\
$A_{2}$ & $A_{1}^{\prime}$ & 1 & 1 & 1 & 1 & 1 & 1 \\
$B_{1}$ & $A_{2}^{\prime}$ & 1 & 1 & 1 & 1 & -1 & -1 \\
$B_{2}$ & $A_{1}^{\prime \prime}$ & 1 & -1 & 1 & -1 & -1 & -1 \\
$E_{2}$ & $A_{2}^{\prime \prime}$ & 1 & -1 & 1 & -1 & 0 & 1 \\
$E_{1}$ & $E^{\prime}$ & 2 & 2 & -1 & -1 & 0 & 0 \\
\hline
\end{tabular}

may expect that representation $E_{1 g}$, as being more symmetrical, is realized at the top of the valence band, and the representation $E_{2 u}$ is realized at the bottom of the conduction band.

To get the form of the energy spectrum of the electrons in the vicinity of the point $\tilde{\Gamma}$ let us consider both the $\boldsymbol{k} \cdot \boldsymbol{p}$ term [17] and the supercell potential as a perturbation. The effective Hamoltonian is

$$
\hat{H}_{\text {eff }}=\hat{H}_{k \cdot p}+\hat{V},
$$

where

$$
\hat{H}_{k \cdot p}=\left(\begin{array}{cccc}
0 & v\left(k_{x}-i k_{y}\right) & 0 & 0 \\
v\left(k_{x}+i k_{y}\right) & 0 & 0 & 0 \\
0 & 0 & 0 & v\left(k_{x}+i k_{y}\right) \\
0 & 0 & v\left(k_{x}-i k_{y}\right) & 0
\end{array}\right),
$$

and $\hat{V}^{(0)}$ reduces to two independent real constants

$$
\hat{V}=\hat{V}^{(0)}=\left(\begin{array}{cccc}
0 & 0 & V_{1} & 0 \\
0 & 0 & 0 & V_{2} \\
V_{1} & 0 & 0 & V_{2} \\
0 & V_{2} & 0 & 0
\end{array}\right) .
$$

The specific form of the operator $\hat{V}^{(0)}$ follows from the symmetry of the base functions realizing representations $E_{1 g}$ and $E_{2 u}$. By shifting origin of the energy axis these two constants can be chosen as

$$
V_{1}=-V_{2}=V .
$$

Forming and solving the secular equation from these matrix elements, we obtain

$$
\varepsilon^{(0)}(k)= \pm \sqrt{v^{2} k^{2}+V^{2}}
$$

the sign plus corresponding to an upper pair of bands, and the sign minus corresponding to a lower pair of bands.

To resolve between the branches in each pair we should take into account $\boldsymbol{k}$ corrections to the operator $\hat{V}^{(0)}$. The first order in $\boldsymbol{k}$ corrections is equal to zero because the symmetry group contains the center of inversion. To the second order in $\boldsymbol{k}$ we have $(i, k=1,2)$

$$
\hat{V}=\hat{V}^{(0)}+\hat{V}^{(2)}=\hat{V}^{(0)}+\hat{\gamma}_{i k} k_{i} k_{k},
$$

where $\hat{\gamma}_{i k}$ is an Hermitian tensor operator (symmetrical in the suffixes $i$ and $k$ ). These include the corrections from the terms linear in $\boldsymbol{k}$ in the Hamiltonian in the second-order perturbation theory and the corections from the terms quadratic in $\boldsymbol{k}$ in the first-order perturbation theory [17]. Notice that $\hat{V}^{(2)}$ is small relative to both $\hat{V}^{(0)}$ (because we consider the states in the vicinity of the point $\tilde{\Gamma}$ ), and with respect to $\hat{H}_{k \cdot p}$.

The relations exist between the matrix elements of the operator because of the requirements of symmetry. As regards their transformation law under the symmetry operations, the wave functions which form the basis of the 
representation $E_{2 u}$ (1, 2 branches) can be taken in the form

$$
\psi_{1} \sim z x, \quad \psi_{2} \sim z y .
$$

and the wave functions which form the basis of the representation $E_{2 u}$ can be taken in the form

$$
\psi_{1} \sim z, \quad \psi_{2} \sim z x y .
$$

From this, we easily conclude that in the first case the matrix elements of the $\hat{\gamma}_{i k}$ reduce to three independent real constants

$$
\begin{aligned}
& \left\langle 1\left|\gamma_{x x}\right| 1\right\rangle=\left\langle 2\left|\gamma_{y y}\right| 2\right\rangle=A, \\
& \left\langle 2\left|\gamma_{x x}\right| 2\right\rangle=\left\langle 1\left|\gamma_{y y}\right| 1\right\rangle=B, \\
& \left\langle 1\left|\gamma_{x y}\right| 2\right\rangle=\left\langle 2\left|\gamma_{x y}\right| 1\right\rangle=C .
\end{aligned}
$$

The matrix elements of the operator $\hat{V}^{(2)}$ are

$$
\begin{aligned}
& \left\langle 1\left|\hat{V}^{(2)}\right| 1\right\rangle=\left\langle 2\left|\hat{V}^{(2)}\right| 2\right\rangle=A k_{x}^{2}+B k_{y}^{2} \\
& \left\langle 1\left|\hat{V}^{(2)}\right| 2\right\rangle=\left\langle 2\left|\hat{V}^{(2)}\right| 1\right\rangle=2 C k_{x} k_{y} .
\end{aligned}
$$

In the second case the matrix elements of the $\hat{\gamma}_{i k}$ also reduce to three independent real constants

$$
\begin{aligned}
& \left\langle 1\left|\gamma_{x x}\right| 1\right\rangle=\left\langle 1\left|\gamma_{y y}\right| 1\right\rangle=D, \\
& \left\langle 2\left|\gamma_{x x}\right| 2\right\rangle=\left\langle 2\left|\gamma_{y y}\right| 2\right\rangle=E, \\
& \left\langle 1\left|\gamma_{x x}\right| 2\right\rangle=\left\langle 1\left|\gamma_{y y}\right| 2\right\rangle=F .
\end{aligned}
$$

The matrix elements of the operator $\hat{V}^{(2)}$ are

$$
\begin{aligned}
& \left\langle 1\left|\hat{V}^{(2)}\right| 1\right\rangle=D k^{2}, \\
& \left\langle 2\left|\hat{V}^{(2)}\right| 2\right\rangle=E k^{2}, \\
& \left\langle 1\left|\hat{V}^{(2)}\right| 2\right\rangle=\left\langle 2\left|\hat{V}^{(2)}\right| 1\right\rangle=2 F k_{X} K_{Y} .
\end{aligned}
$$

Forming and solving the secular equation from these matrix elements, we obtain the $E_{1 q}$ branches of the spectrum

$$
\varepsilon(\boldsymbol{k})=\varepsilon^{(0)}(\boldsymbol{k})+A k_{x}^{2}+B k_{y}^{2} \pm 2 C k_{x} k_{y}
$$

The sign of $\varepsilon^{(0)}(\boldsymbol{k})$ is determined by the fact whether we are dealing with the lower or upper bands. The formula for the $E_{2 u}$ branches of the spectrumcan is obtained similarly.

The folding of the BZ, together with the destruction of previously existing gapless Dirac points, leads to appearance of the new ones. In fact, the new BZ is still a hexagon, and the same symmetry arguments used for graphene can be used to explain appearance of the gapless Dirac points at the corners of the new BZ $\left(\tilde{\boldsymbol{K}}, \tilde{\boldsymbol{K}}^{\prime}\right)$. However, these new Dirac points are situated deep below or high above the Fermi level, hence, less of them manifest themselves than Dirac points of unreconstructed graphene.

\section{References}

[1] Uchoa, B., Lin, C.-Y. and Castro Neto, A.H. (2008) Tailoring Graphene with Metals on Top. Physical Review B, 77, Article ID: 035420.

[2] Giovannetti, G., Khomyakov, P.A., Brocks, G.V., Karpan, M., van den Brink, J. and Kelly, P.J. (2008) Doping Graphene with Metal Contacts. Physical Review Letters, 101, Article ID: 026803.

[3] Elias, D.C., Nair, R.R., Mohiuddin, T.M.G., Morozov, S.V., Blake, P., Halsall, M.P., Ferrari, A.C., Boukhvalov, D.W., Katsnelson, M.I., Geim, A.K. and Novoselov, K.S. (2009) Control of Graphene's Properties by Reversible Hydrogenation: Evidence for Graphene. Science, 323, 610-613. http://dx.doi.org/10.1126/science.1167130

[4] Chen, J.-H., Jang, C., Adam, S., Fuhrer, M.S., Williams, E.D. and Ishigami, M. (2008) Charged-Impurity Scattering in Graphene. Nature Physics, 4, 377-381. http://dx.doi.org/10.1038/nphys935

[5] Zhou, S.Y., Siegel, D.A., Fedorov, A.V. and Lanzara, A., (2008) Metal to Insulator Transition in Epitaxial Graphene Induced by Molecular Doping. Physical Review Letters, 101, Article ID: 086402. 
http://dx.doi.org/10.1103/PhysRevLett.101.086402

[6] Schedin, F., Geim, A.K., Morozov, S.V., Hill, E.W., Blake, P., Katsnelson, M.I. and Novoselov, K.S. (2013) Detection of Individual Gas Molecules Adsorbed on Graphene. Nature Materials, 6, 652-655. http://dx.doi.org/10.1038/nmat1967

[7] Lamoen, D. and Persson, B.N.J. (1998) Adsorption of Potassium and Oxygen on Graphite: A Theoretical Study. Journal of Chemical Physics, 108, 3332-3341. http://dx.doi.org/10.1063/1.475732

[8] Vasseur, G., Fagot-Revurat, Y., Kierren, B., Sicot, M. and Malterre, D. (2013) Effect of Symmetry Breaking on Electronic Band Structure: Gap Opening at the High Symmetry Points. Symmetry, 5, 344-354. http://dx.doi.org/10.3390/sym5040344

[9] Farjam, M. and Rafii-Tabar, H. (2009) Energy Gap Opening in Submonolayer Lithium on Graphene: Local Density Functional and Tight-Binding Calculations. Physical Review B, 79, Article ID: 045417.

[10] Kogan, E. and Nazarov, V.U. (2012) Symmetry Classification of Energy Bands in Graphene. Physical Review B, 85, Article ID: 115418. http://dx.doi.org/10.1103/PhysRevB.85.115418

[11] Kogan, E. (2013) Symmetry Classification of Energy Bands in Graphene and Silicene. Graphene, 2, 74-80. http://dx.doi.org/10.4236/graphene.2013.22011

[12] Kogan, E., Nazarov, V.U., Silkin, V.M. and Kaveh, M. (2013) Energy Bands in Graphene: How good Is the TightBinding Model? Physical Review B (Submitted for Publication).

[13] Bradley, C.J. and Cracknell, A.P. (1972) The Mathematical Theory of Symmetry in Solids. Clarendon Press, Oxford.

[14] Aroyo, M.I., Capillas, C., De la Flor, G., Kirov, A.K., Orobengoa, D., Perez-Mato, J. and Wondraschek, H. (2010) Representations of Cristallographic Groups. http://www.crystallography.fr/mathcryst/pdf/nancy2010/Aroyo reps2010.pdf

[15] Cheianov, V.V., Falko, V.I., Syljyuasen, O. and Altshuler, B.L. (2009) Hidden Kekulé Ordering of Adatoms on Graphene. Solid State Communications, 149, 1499-1501. http://dx.doi.org/10.1016/j.ssc.2009.07.008

[16] Landau, L.D. and Lifshitz, E.M. (1991) Landau and Lissitz: Course of Theoretical Physics on Quantum Mechanics. Pergamon Press, Oxford.

[17] Lifshitz, E.M. and Pitaevskii, L.P. (1979) Landau and Lissitz: Course of Theoretical Physics on Statistical Physics. Pergamon Press, Oxford. 